\title{
618503
}

\section{SOME IMPLICATIONS OF SATELLITE SPIN EFFECTS IN CYLINDRICAL PROBE MEASÚREMENTS}

\section{NATHAN J.MILLER}

NOVEMBER 1971

\section{GREENBELT, MÁRYLAND}

(NASA-TM-X-65803) SOHE IMPLICATIONS OF

HEASUREUENTS N. SFETS IN CYLINDRICAL PROBE

$34 p$

N.J. Miller (NASA)

NOV. 1971

CSCL $22 \mathrm{C}$
RATIIONAL TECHNICAL

INFORMATION SERVICE

US Dopartment of Commerce
Springliald, VA. 22151

N 72-16747

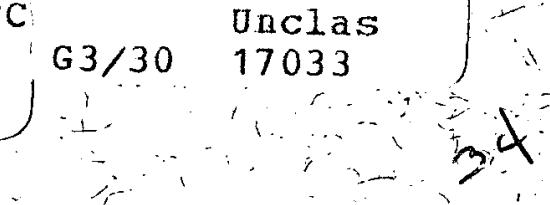


$\mathrm{X}-621-71-446$

Preprint

\section{SOME IMPLICATIONS OF SATELLITE SPIN EFFECTS}

IN CYLINDRICAL PROBE MEASUREMENTS

Nathan J. Miller

November 1971

GODDARD SPACE FLIGHT CENTER

Greenbelt, Maryland 


\title{
PCFDING PAGE BLANK NOT FILIVED \\ SOME IMPLICATIONS OF SATELLITE SPIN EFFECTS
}

IN CYLINDRICAL PROBE MEASUREMENTS

Nathan J. Miller

\begin{abstract}
In-situ measurements of ambient electron densities. with satellite-borne cylindrical probes exhibit periodic variations synchronous with the satellite's spin cycle. Representing these fluctuations as a superposition of effects attributable to both the presence of the satellite wake and the geomagnetic field leads to a model of the modulations of accelerated electron current to cylindrical probes in which one modulation component displays current variations dependent upon the probe-velocity angle $(\psi)$ and the other displays variations dependent upon the angle between the probe axis and the geomagnetic field lines ( $\beta$ ). The modulations produce an electron current decrease whenever the probe axis rotates into the satellite wake or whenever the probe axis rotates toward alignment with the geomagnetic field lines. With increasing altitude, the modulation dependent upon $\psi$ decreases whereas the modulation dependent upon $\beta$ increases. The $\psi$ dependent modulation component can be associated with the presence of a wake structure and the $\beta$ dependent component can be associated with magnetic influence on the transport properties of thermal electrons such that electron fluxes are predominately along the field lines. The analysis results
\end{abstract}


imply that the most accurate determinations of atmospheric electron densities by satellite-borne cylindrical probes come from measurements taken out of the satellite's wake and when the probe axis is within $20^{\circ}$ of being perpendicular to the geomagnetic field lines. 


\section{SOME IMPLICATIONS OF SATELLITE SPIN EFFECTS \\ IN CYLINDRICAL PROBE MEASUREMENTS}

\section{INTRODUCTION}

When the attempt is made to measure the density of thermal atmospheric electrons via direct measurement satellite experiments, it is understood that . the satellite may perturb the medium. Consequently, scientists have constructed mathematical models of the charged particle redistribution about satellites so that changes in particle density caused by a satellite's presence can be considered in measurement analysis and in the planning of new experiments (Davis and Harris, 1961; Beard and Johnson, 1965; Al'pert et al., 1965; Walker, 1965; Taylor, 1967a, 1967b; Osborne and Kasha, 1967; Liu, 1969). These models are not necessarily accurate descriptions of the true ionospheric-satellite interaction but they serve as first steps in studies which can be corrected, on the basis of experimental measurements, to produce increasingly more realistic models.

Langmuir probe data has been found to contain periodic electron current variations in synchronization with a satellite's spin cycle (Bourdeau and Donley, 1965; Samir and Willmore, 1965; Henderson and Samir, 1967; Wrenn, 1969). The results were not unanticipated since a planar Langmuir probe whose collecting surface is oriented at $90^{\circ}$ to a satellite's spin axis is expected to detect electron density variations as a direct consequence of the probe's rotation through the satellite's wake structure. Additional causes of periodic 
probe current fluctuations have also been investigated. The relationship between the orientation of a moving cylindrical probe and its ion current collection was analyzed mathematically by Kanal (1964). He constructed theoretical volt-ampere curves for different probe-velocity angles and found that the orientation had negligible effect upon the electron temperature determination, but had an increasingly important effect upon the accelerated ion current as the ratio of probe velocity to the most probable ion speed increased. As a further refinement, several authors (Bettinger and Chen, 1968; Hester and Sonin, 1970) deduced the effects of ion current collected through the end of a satellite borne cylindrical probe and showed that their predictions were qualitatively in line with experimental measurements of increased ion currents when the probe is in the ram position. A directionally sensitive instrument such as a satellite borne cylindrical probe might also detect spin modulations in the electron current collection because the geomagnetic field creates a preferred direction of transport along the field lines for thermal electrons.

This report discusses cylindrical Langmuir probe measurements of accelerated electron current taken during the winter 1965-66. According to Langmuir probe theory, the current measurements are directly proportional to $\mathrm{N}_{\mathrm{e}}$ in the ambient plasma. Under some conditions, the electron densities determined from such data may vary by factors of 2 or 3 because of the spin modulations. Measurements from at least 500 satellite rotations were studied in order to get an understanding of these modulations. The specific examples 
chosen to represent the data came from geomagnetic latitudes between $-12^{\circ}$ and $-45^{\circ}$. The measurements contain the effects of both the satellite motion through the plasma and the magnetic effects on electron thermal motions in a medium permeated by a magnetic field. The modulating influences act simultaneously, so a heuristic model of current modulations is used to separate the individual influences and to investigate their geophysical implications.

\section{THE EXPERIMENT}

The experimental results come from the cylindrical Langmuir probe experiment aboard the Explorer 31 satellite, a right octagonal prism (Figure 1). The satellite spin axis is along the satellite's axis of symmetry and is generally perpendicular to the orbital plane. The Langmuir probe experiment consists of two electrostatic probes mounted at $90^{\circ}$ to the satellite's spin axis and positioned on opposite sides of the satellite near one end. The diameter of the probes is approximately $0.06 \mathrm{~cm}$ as compared to the mean gyro radius for electrons of 3 to $14 \mathrm{~cm}$ for the altitude range of $500-3000 \mathrm{~km}$ where the satellite is operating. The distance between opposing side panels of the satellite is $75 \mathrm{~cm}$ and the probe collector surface spans the range $23-46 \mathrm{~cm}$ outward from a satellite panel.

A sawtooth voltage is independently applied to each probe at a frequency that generates about 18 volt-ampere curves per spin cycle of 20 seconds. During a rotation, the volt-ampere curves associated with one probe have a current resolution suited to electron density $\left(\mathrm{N}_{\mathrm{e}}\right)$ analysis, which employs accelerated electron current, and the other volt-ampere curves are suited to electron 
- temperature analysis, which employs retarded electron current. The curves appropriate for $\mathrm{N}_{\mathrm{e}}$ analysis display both a variation dependent upon the probevelocity angle and a variation dependent upon the probe's orientation to the geomagnetic field. The volt-ampere curves used for electron temperature analysis display little or no spin modulation except for the variations in ion current as discussed by Brace, et al. (1971). Since this report is only concerned with systematic modulations which appear in the accelerated electron currents, the reader is referred to the literature for specifics on the experimental electronics, measurement theory, and the probe equations associated with the Langmuir probe experiment (Brace and Findlay, 1969; Findlay and Brace, 1969; Mott-Smith and Langmuir, 1926).

THE DATA

Figure 2 shows volt-ampere curves from the Explorer 31 probe experiment that are typical for those data used in $\mathrm{N}_{\mathrm{e}}$ analysis. The base portion of the current curves is the electron retarding region. The ascending portions of the current curves represent accelerated electron current, which is drawn when the probe becomes positive relative to the plasma. Variations in the curve heights are the result of the spin modulations. An envelope of the high voltage endpoints of the current curves approximates a continuous time variation in accelerated electron current that would occur at constant positive probe potential. Curve $A$ in Figure 3 shows how such an envelope looks when, instead of time, the probe-v locity angle $(\psi)$ is used as abscissa; $\psi$ is the angle between 
the probe axis and the satellite's velocity vector. The data were taken at an altitude of $770 \mathrm{~km}$ over a single spin cycle which spans $160 \mathrm{~km}$ along the orbit path. Overlap in current values between the first and the last data points of the spin cycle was close enough to assure that changing atmospheric conditions were a minor influence on the current variations observed within a single spin cycle. The overlap criterion was used throughout this study as a standard in deciding which sets of data were least affected by spatial gradients or time variations in the electron density and therefore would display the clearest examples of the spin modulation.

Envelope curve A has approximate symmetry about the direction of the satellite velocity. Curve B represents the same data plotted against a field angle $\beta$, the angle between the probe axis and the geomagnetic field. With respect to field line orientation, curve B did not display an obvious symmetry. A more compact method for plotting envelope curves is to use an abscissa with angular values $0^{\circ}$ to $180^{\circ}$ and connect the data points in the order of their time sequence. In Figure 4, which contains data taken near $600 \mathrm{~km}$, such an envelope curve is drawn with arrows along the curve to indicate the order in which the data was measured. The compact plotting form causes any degree of asymmetry about the velocity vector or the field direction to result in a loop structure for the envelope curve. The spin modulation displayed in Figure 4 is nearly symmetric about the velocity vector as was the data of Figure 3. 
At higher altitudes, the probe measurements contain a greater degree of geomagnetic field influence; thus, in Figure 5 there is near symmetry about the direction of the geomagnetic field with current minima occurring whenever the probe axis lies along the field lines. Current decreases occurred for a similar orientation in Figure 3 but the deep wake made this feature less obvious. The polar plot of Figure 6 shows the angular distribution of the data of Figure 5 in a manner that more dramatically illustrates the magnetic field influence. Figure 7 is a polar plot of data taken at a low altitude where the decrease in density in the wake is more significant than at a high altitude. Here the geomagnetic field influence leads to an asymmetric wake. In Figures 6 and 7 the probe axis passed within $10^{\circ}$ of both the field line and the satellite velocity directions, so the plane of the graph represents the plane in which the measurements were taken.

An investigation of a large amount of Explorer 31 probe data makes it clear that the loop form of envelope curves in Figures 4 and 5 was not merely the result of random experimental errors in current measurements. Using the . compact plotting form, additional samples of data from altitudes between $600 \mathrm{~km}$ and $2000 \mathrm{~km}$ are shown in Figures 8 and 9. These plots illustrate the extent to which changing the independent variable affects the form of the envelope curve. In general, the illustrated data represents cases where the satellite spin axis was nearly perpendicular to the plane formed by unit vectors in the direction of $\overrightarrow{\mathrm{v}}$ and $\overrightarrow{\mathrm{B}}$. This condition allowed the probe sixis to pass near both the satellite velocity direction and the magnetic field direction, making it possible to study 
measurements made over the maximum range of both probe-velocity and field angles. In most of the data studied, the directions of $\vec{v} \times \vec{B}$ and the satellite axis were not parallel so the data to which a detailed analysis is applied were special cases chosen for ease of analysis and because they would provide the best examples of the two principal modulation components whose effects appeared in all of the data. The envelope curves of Figures 4 and 5 were presented separately because those current modulations appeared to be predominately dependent upon a single modulation component, a condition which made it possible to see the general angular dependence of one modulating influence by plotting against a single variable. In most cases, the total modulation was dependent upon both $\psi$ and $\beta$; hence, it was necessary to make physical assumptions in order to decompose the total current modulation and obtain a quantitative estimate of the magnitude of the modulation component.

\section{THE SUPERPOSITION MODEL}

Though an envelope of the end-points on the volt-ampere curves can be drawn, no information exists between these points to suggest how the most representative envelope should be constructed; thus, a current envelope curve must be made consistent with reasonable physical assumptions. The first assumption was that the total modulation is the superposition of two independent components, one only $\psi$ dependent, $\Delta \mathrm{J}(\psi)$, and the other only $\beta$ dependent, $\Delta \mathrm{J}(\beta)$. The magnitude of the total modulation, $\Delta \mathrm{J}(\psi, \beta) \equiv \Delta \mathrm{J}(\psi)+\Delta \mathrm{J}(\beta)$, was 
taken as the difference in value between the measured current and some standard current, e.g., the maximum current measured during a spin cycle might be taken as the standard current. A second assumption was that the separate modulation components should be symmetrical about some basic position. This required that $\Delta \mathrm{J}(\psi)$ should have angular symmetry about the direction of the satellite velocity. On Figure 4, a $\psi$ dependent modulating influence would result in equal current collection at both the primed and unprimed probe orientations. The fact that different currents are measured at similar $\psi$ orientations is taken as evidence for a $\beta$ dependent component in the total modulation. In this way, the lack of complete symmetry in the envelope curve $A$ in Figure 3 is considered to be due to the magnetic component of the total modulation. A symmetry assumption was also made concerning $\Delta \mathrm{J}(\beta)$. In this case, $\Delta \mathrm{J}(\beta)$ was assumed to have angular symmetry about the geomagnetic field lines. In Figure $5, \Delta \mathrm{J}(\beta)$ would lead to equal current collection at both primed and unprimed probe orientations. That this does not occur in the total current measurements is attributed to the influence of the satellite wake structure as previously discussed. Finally, it was assumed that the wake structure does not interfere with current collection in the forward direction for $\psi \leq 50^{\circ}$. This assumption allowed current decreases in the forward direction to be assigned as values of $\Delta \mathrm{J}(\beta)$ for the purposes of calculations.

To begin the analysis, a smooth curve was drawn through each set of data taken over a complete satellite rotation just as was done in Figures 3-9. Using 
the given assumptions, deviations from a standard current value were defined as the magnitude of $\Delta \mathrm{J}(\psi, \beta)$, where the standard current for each curve was chosen to be the maximum accelerated electron current measured over a satellite rotation. Since it was assumed that current deviations in the forward direction.were only $\beta$ dependent, the magnitude of current decreases for orientations . with $\psi \leq 50^{\circ}$ were defined as values of $\Delta \mathrm{J}(\beta)$ and used to deduce the remaining values for $\Delta \mathrm{J}(\beta)$ at other field angles. Values for $\Delta \mathrm{J}(\psi)$ were calculated as the difference between the values of $\Delta J(\psi, \beta)$ and $\Delta J(\beta)$. The separate normalized modulation components determined by applying the model assumptions to the raw data presented in this report are shown in Figures 10 and 11. Since a very simplified model is used as a basis for analysis, the fine structure of the calculated $\Delta \mathrm{J}(\psi)$ and $\Delta \mathrm{J}(\beta)$ functions should not be considered when examining the gross features.

\section{DISCUSSION}

\section{Modulations Dependent Upon Probe-Velocity Angle}

In the introductory section, it was stated that one expected source of probe current modulations was the wake structure of the satellite. A charged particle wake forms because the satellite velocity is greater than the mean thermal velocity for the ions so the ions are unable to rapidly fill in behind a satellite as it passes through the topside plasma. The electrons are then constrained from completely filling in the wake by attraction to the ions outside the wake and by repulsion from electrons already in the wake. Near the satellite surface, 
Gurevich, et al. (1964) refer to a wake region of maximum rarefaction for angles to the satellite velocity greater than $120^{\circ}$. A charged satellite is surrounded by a plasma sheath but in the angular range of the region of maximum rarefaction, the limited ability of the ions to fill in the wake creates an abnormally large region of unbalanced charge, which can be physically described as a plasma sheath. In this region, both electron and ion densities are attenuated beyond the degree of depletion in other wake regions.

Experimental measurements in the wake by Samir and Wrenn (1969) displayed a decrease in the depth of the charged particle wake at high altitudes. Their determinations of ion composition and electron currents from two experiments aboard Explorer 31 were consistent with the assumption that a decreasing electron wake depth is caused by changes in principal ion from, $\mathrm{O}^{+}$to $\mathrm{H}^{+}$. Another wake feature was hinted at by Samir and Willmore (1965) who showed a curve displaying the average angular variation in ion current collection based on probe data taken over many satellite rotations. This curve suggested an ion wake enhancement near $120^{\circ}$ relative to the satellite velocity direction.

In the format used to present the results of this report $\Delta J(\psi)$ represents the fractional decrease in electron current collection when only changes in probe-velocity angle are considered. When comparisons are made between what is displayed by the modulation dependent upon probe-velocity angle and what was observed in previous wake studies the form of $\Delta \mathrm{J}(\psi)$ adds to the confidence that the superposition analysis has produced a reasonable separation of modulation 
components. The curves of $\Delta J(\psi)$ in Figure 10 exhibit an altitudinal trend of decreasing depth at high altitudes and some examples of wake enhancements. The modulation changed from a $60 \%$ current reduction in the wake at $600 \mathrm{~km}$ to a $30 \%$ reduction at $2200 \mathrm{~km}$. The position where a significant decrease in $\Delta \mathbf{J}(\psi)$ begins can be interpreted as the angular boundary for the region of maximum rarefaction at the probe distance from the satellite surface. The undecomposed data for curve $\mathrm{A}$ in Figure 3 supports the conclusion drawn from the superposition analysis that the wake enhancement is $\psi$ dependent since curve A is an example where the wake enhancement in the raw data can be seen to have an approximate symmetry about the velocity vector. The wake enhancement is not always present and when it is, the geomagnetic influence in the total modulation can make the symmetry less obvious. The enhancement at $120^{\circ}$ in the $\Delta \mathrm{J}(\psi)$ curves can be considered as evidence for an electron effect, generated by the passage of the satellite through the plasma, which has not been accounted for in the theories of satellite-ionosphere interactions.

\section{Modulations Dependent Upon the Field Angle}

In experimental measurements, a dependence upon magnetic orientation is expected to be only obvious in the data when the measurements involve a scale length larger than the mean gyro radius. Thus, one would postulate that measurements of electron current employing a cylindrical probe whose radius is much less than the mean electron gyro radius and which collects particles from all pitch angles would contain practically no evidence of a magnetic orientation 
effect. However, the modulation curves of $\Delta \mathrm{J}(\beta)$ exhibit a definite decrease in probe current whenever the probe axis passes parallel to the field lines. This experimental result suggests that the magnetic field may be restricting the current collection. When the probe axis is within $20^{\circ}$ of the perpendicular to the field lines, the fractional current decrease is negligible. The magnetic component of the current modulation produces the largest current decreases at the highest altitudes and since the depth of the electron wake is least there, the magnetic influence becomes more important than the wake effects.

A physical explanation of the magnetic modulations can be arrived at by examining the probe's current collecting mechanism. The probe draws its current from the charged particles which enter its plasma sheath. At equilibrium, the sheath boundary for a positive probe must encompass a volume of plasma that contains enough electrons to neutralize the positive probe potential. The effect of a decreasing plasma density at constant probe potential is to cause a sheath expansion in order to maintain a constant amount of negative charge within the sheath volume. When the sheath radius is much larger than a mean gyro radius a probe oriented parallel to the field must collect a substantial portion of its current by attracting electrons across field lines. In contrast, a probe oriented perpendicular to the field can collect electron current by attracting electrons along the field lines. Within this mechanism one expects any magnetic orientation dependence in the current collection process to be related to the difference in electron transport parallel and perpendicular to 
the field. If the sheath radius grows relative to the mean gyro radius the modulation should become more obvious.

As an illustrative example, the scale length concept can be applied to the Passes 776 and 1408 in Figure 11 which shows different degrees of magnetic modulation. The atmospheric parameters which differed significantly between the two passes were the electron temperature and the magnetic field strength. An actual sheath size is difficult to calculate for a moving probe, but sheath radius is connected with Debye length. The ratios of Debye lengths and electron gyro radii in these two passes are 1.3 and 2.3 respectively, where both length parameters are greater in Pass 1408. The calculated length ratios suggest that the gyro radius increased more than the sheath size and therefore, the modulation should be less in Pass 1408. The curves of $\Delta \mathrm{J}(\beta)$ are consistent with this conclusion. The ratio comparison is only used to obtain a qualitative estimate of the effect that relative changes between sheath radius and mean electron gyro radius might have in changing the depth of the magnetic modulation. However, the calculation suggests that the apparent altitudinal trend in the depth of the magnetic modulation is really caused by a changing relationship between the gyro radius and sheath size. Ultimately, a theoretical description of current collection by the cylindrical probe in a magnetized plasma is needed in order to fully explain the magnetic modulation effects. 


\section{Other Influences}

It is understood that influences other than those previously discussed in detail can affect the modulation of accelerated electron current, e.g., changes in satellite potential or $\overrightarrow{\mathrm{v}} \times \overrightarrow{\mathrm{B}}$ effects on the probe. Individually, these other. influences were either not of sufficient magnitude or would not produce the kind of variation in electron current required for the continuous periodic modulations with which this report has been concerned. The accelerating probe potential which results in the collection of electron current is defined relative to the plasma potential. On the other hand, the applied probe potential is produced relative to the satellite potential. The result is that changes in space potential affect the collection of accelerated electron current. Samir and Wrenn (1969) gave experimental examples of the variations in space potential which can occur about a spacecraft. In the present study, these space potential variations did not correlate with the current modulations and were too small to produce the degree of current change that was observed.

Another effect in the current data exists because of the finite length of the probe. In this case, the electron current sometimes decreases as the probe axis passes through the velocity ram position as in curve A of Figure 3 . Since one of the model assumptions was that there were no velocity orientation effects for $\psi \leq 50^{\circ}$, those passes which contained obvious electron current end effects were not used for the detailed analysis. 
The overlap criterion which required repetition within the data between consecutive satellite passes reduced the effect of gradients or temporal variations in the density which would affect the current modulations. Finally, the effects of any induced potential from the movement of the satellite across magnetic field lines was reduced by choosing satellite rotations where the vector $\vec{v} \times \vec{B}$ was essentially parallel to the spin axis of the satellite. In this manner, the probe axis was rotating in a plane of nearly constant induced potential.

\section{CONCLUSIONS}

A heuristic approach to the study of current modulations within the accelerated electron current measured via the cylindrical probe experiment aboard Explorer 31 suggests that the total modulation can be analyzed as a linear superposition of probe-velocity angle $(\psi)$ and probe-field line angle $(\beta)$ dependent modulations. This assumption along with that of symmetry permitted the general behavior of persistent periodic electron current modulations to be displayed and interpreted in a simplified manner. The $\psi$ dependent modulation contained a decrease of current in the satellite wake similar in magnitude to that detected by other researchers and also occasionally displayed an electron current enhancement which appeared near $120^{\circ}$. The $\beta$ dependent modulation contained current decreases whenever the probe axis approached the field line direction. The altitude trends of the two types of modulations ran directly counter, with the $\psi$ dependent modulation decreasing at higher altitudes. This trend makes the magnetic modulation of probe electron current the more important modulation 
component at high altitudes. The modulation $\Delta \mathrm{J}(\psi)$ can be associated with the redistribution of plasma about a moving satellite. The decrease in the magnitude of $\Delta \mathrm{J}(\psi)$ at increasing altitude is consistent with a change in principal ion from $0^{+}$to $\mathrm{H}^{+}$. The modulation $\Delta \mathrm{J}(\beta)$ can be associated with the anisotropic transport of thermal electrons in a geomagnetic field. The changing magnitude of $\Delta \mathrm{J}(\beta)$ may be caused by relative length changes between the sheath radius and the mean electron gyro radius. Of importance to experimenters is the inference that above $600 \mathrm{~km}$, the probe measurements provide the most reliable electron density determinations when the probe axis is simultaneously at a probe-velocity angle of $90^{\circ}$ or less and within $20^{\circ}$ of being transverse to the geomagnetic field lines.

\section{ACKNOWLEDGEMENTS}

The author thanks L. H. Brace and Drs. J. Grebowsky, W. Hoegy and R. Hartle for helpful discussions. Also acknowledged is the cooperation of L. Rudolf and E. Gregg in providing data in a usable form. 


\section{REFERENCES}

Al'pert, Y. L., A. V. Gurevich, L. P. Pitaevskii, Space Physics With Artificial Satellites, Consultants Bureau, New York, 1965.

Beard, D. B. and F. S. Johnson, Charge and magnetic field interaction with satellites, J. Geophys. Res., $\underline{65}, 1,1960$.

Bettinger, Richard T. and A. Anthony Chen, An end effect associated with cylindrical Langmuir probes moving at satellite velocities, J. Geophys. Res., $\underline{73}, 2513,1968$.

Bourdeau, R. E. and J. L. Donley, Explorer 8 satellite measurements in the upper ione sphere, Proc. Royal Soc., A281, 285, 1965.

Brace, L. H. and James A. Findlay, Comparison of cylindrical electrostatic probe measurements on Alouette 2 and Explorer 31 satellites, Proc. of the IEEE, 57, 1057, 1969.

Brace, L. H., G. R. Carignan, J. A. Findlay, Evaluation of ionospheric electron temperature measurements by cylindrical electrostatic probes, Space Research, 11, 1079, 1971.

Davis, A. H. and I. Harris, Interaction of a charged satellite with the ionosphere, Rarified Gas Dynamics, Supplement I, ed. by L. Talbot, p. 691, Academic Press, New York, 1961.

Findlay, James A. and L. H. Brace, Cylindrical electrostatic probes employed on Alouette 2 and Explorer 31 satellites, Proc. of the IEEE, 57, 1054, 1969. 
Gurevich, A. V., L. P. Pitaevskii, V. V. Smirnova, Ionospheric aerodynamics, Space Sci. Rev., $\underline{9}, 805,1969$.

Henderson, C. L. and U. Samir, Observations of the disturbed region around an ionospheric spacecraft, Planet. Space Sci., 15, 1499, 1967.

Hester, S. D. and Ain A. Sonin, Ion temperature sensitive end effect in cylindrical Langmuir probe response at ionosphere satellite conditions, Phys. Fluids, $\underline{13}, 1265,1970$.

Kanal, Madhoo, Theory of current collection of moving cylindrical probes, J. Appl. Phys., 35, 1697, 1964.

Liu, Y. C., Ionospheric gas dynamics of satellites and diagnostic probes, Space Sci. Rev., $\underline{9}, 423,1969$.

Mott-Smith, H. M. and Irving Langmuir, The theory of collectors in gaseous discharges, Phys. Rev., 28, 727, 1926.

Osborne, F. J. F. and M. A. Kasha, The $\vec{V} \times \vec{B}$ interaction of a satellite with its environment, Canadian J. Phys., $\underline{45}, 263,1967$.

Samir, U. and A. P. Willmore, The distribution of charged particles near a moving spacecraft, Planet. Space Sci., $\underline{13}, 285,1965$.

Samir, U. and G. L. Wrenn, The dependence of charge and potential distribution around a spacecraft on ionic composition, Planet. Space Sci., 17, 693, 1969. Taylor, J. C., Disturbance of a rarified plasma by a supersonic body on the basis of the Poisson-Vlasov equations-I The heuristic method, Planet. Space Sci., $15,155,1967 \mathrm{a}$. 
Taylor, J. C., Disturbance of a rarified plasma by a supersonic body on the basis of the Poisson-Vlasov equations-II Formal method, Planet. Space Sci., 15, $463,1967 \mathrm{~b}$.

Walker, E. H., Plasma sheath and screening around a stationary charged sphere and a rapidly moving charged body, Interaction of Space Vehicles with an Ionized Atmosphere, ed. by S. F. Singer, p. 61, Pergamon Press, New York, 1965.

Wrenn, Gordon, The Langmuir plate and spherical ion probe experiments aboard Explorer 31, Proceedings of the IEEE, 57, $1072,1969$. 


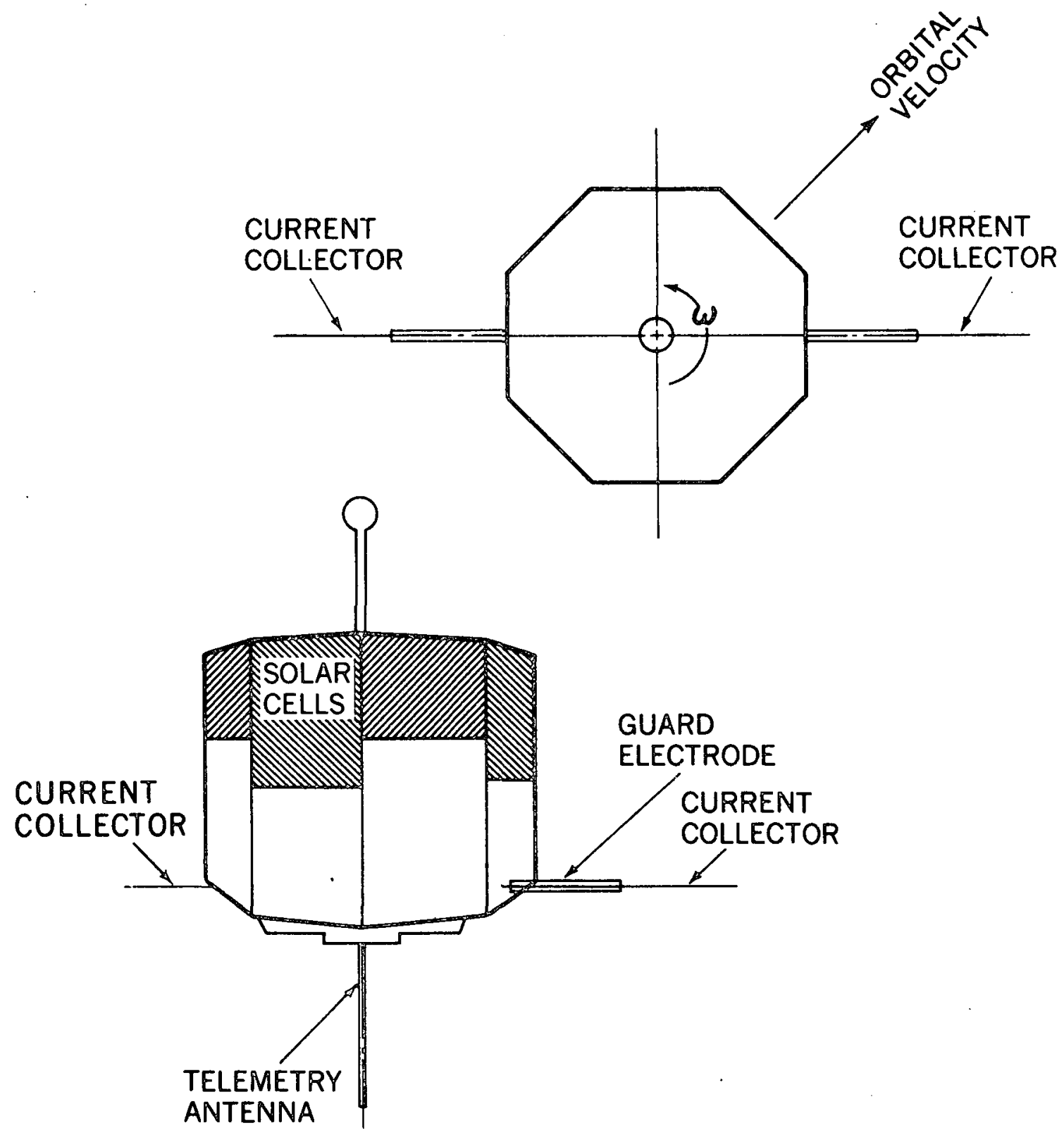

Figure 1. Schemata for Explorer 31 and its Langmuir probe experiment. 


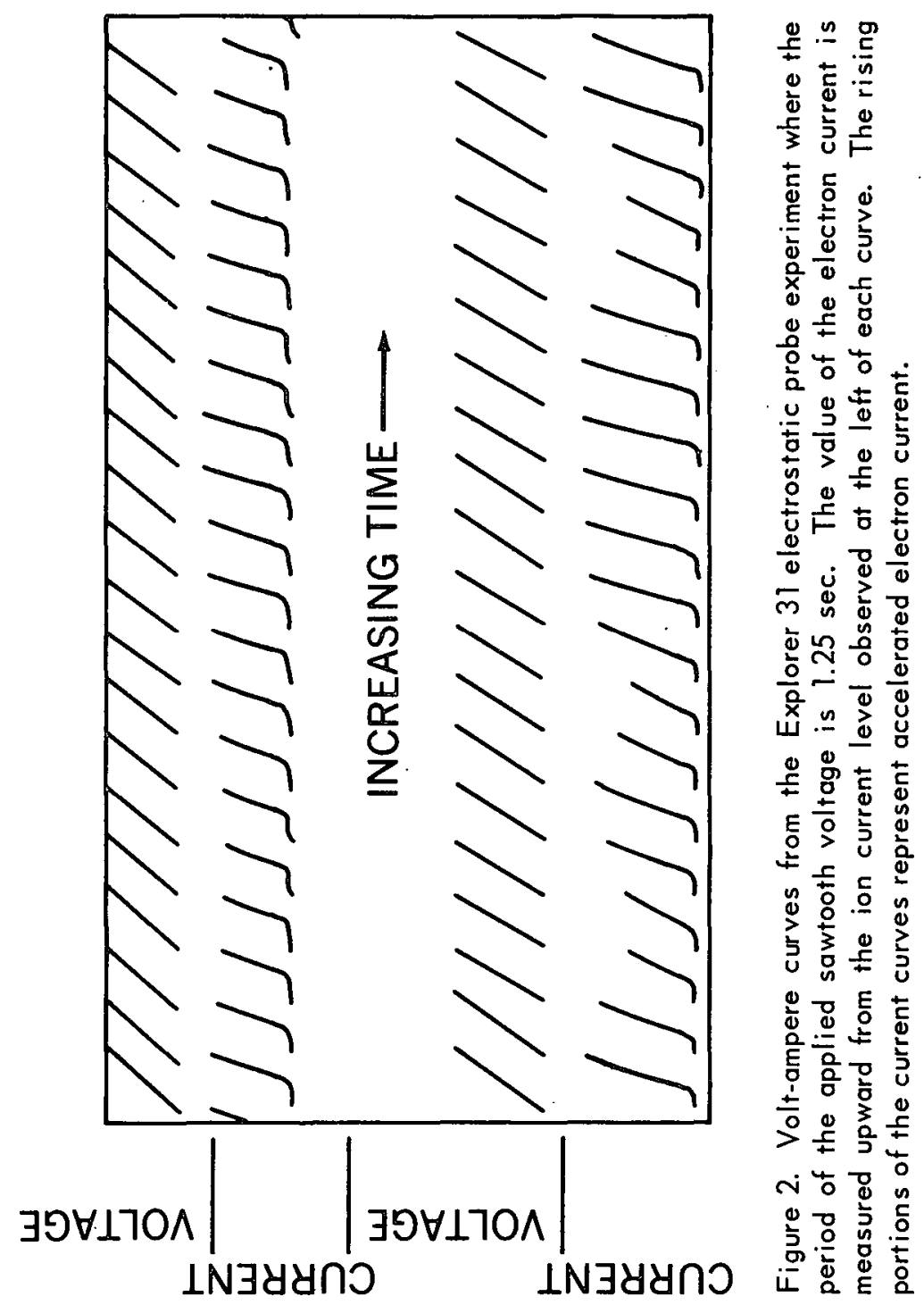




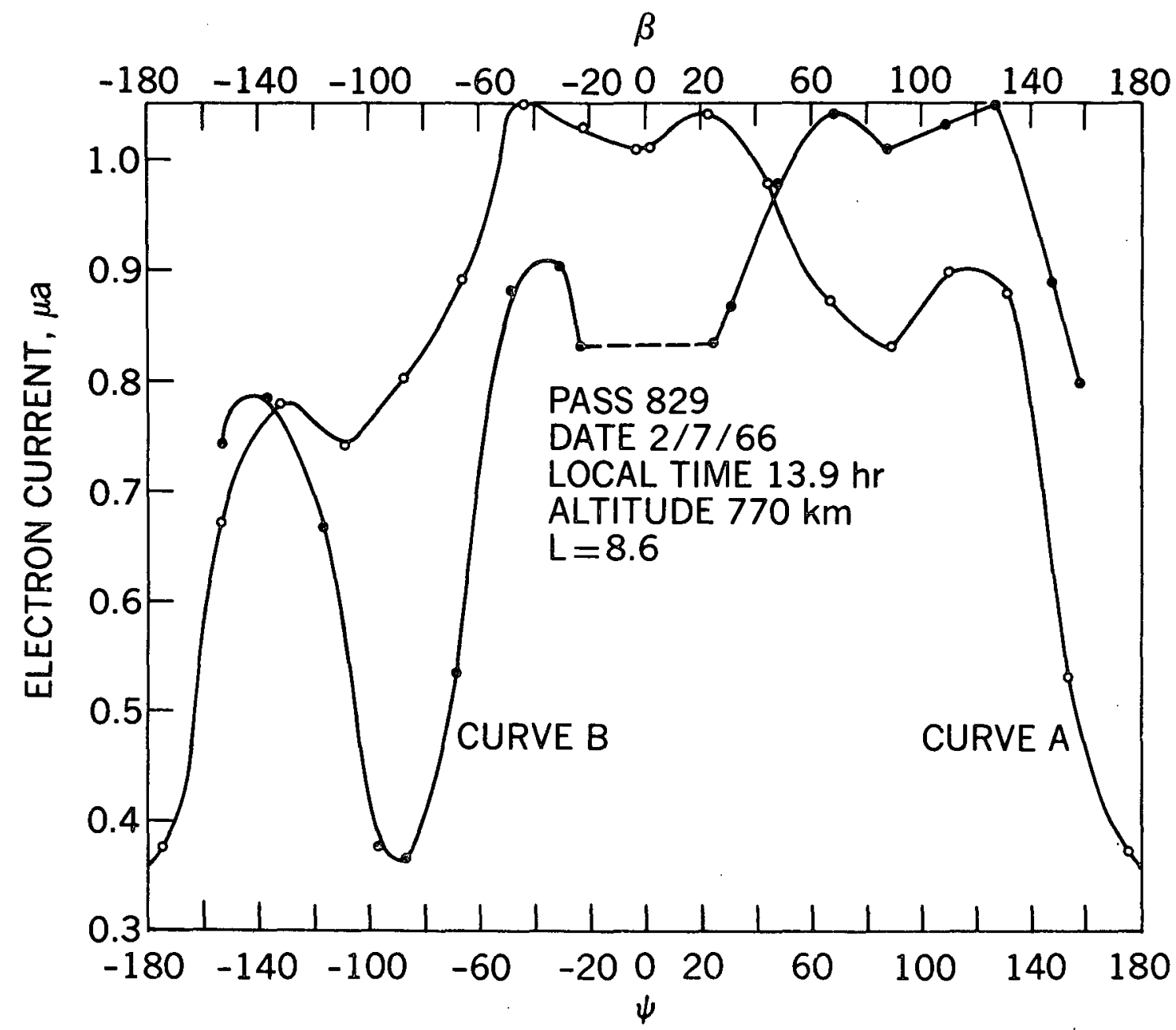

Figure 3. Envelope for a set of volt-ampere curves taken over a single satellite rotation. Curve $A$ is plotted against the probe-velocity angle, $\psi$. Curve $B$ is the same data plotted against the field angle, $\beta$. The minimum angle between the probe axis and the field lines was $22^{\circ}$. 


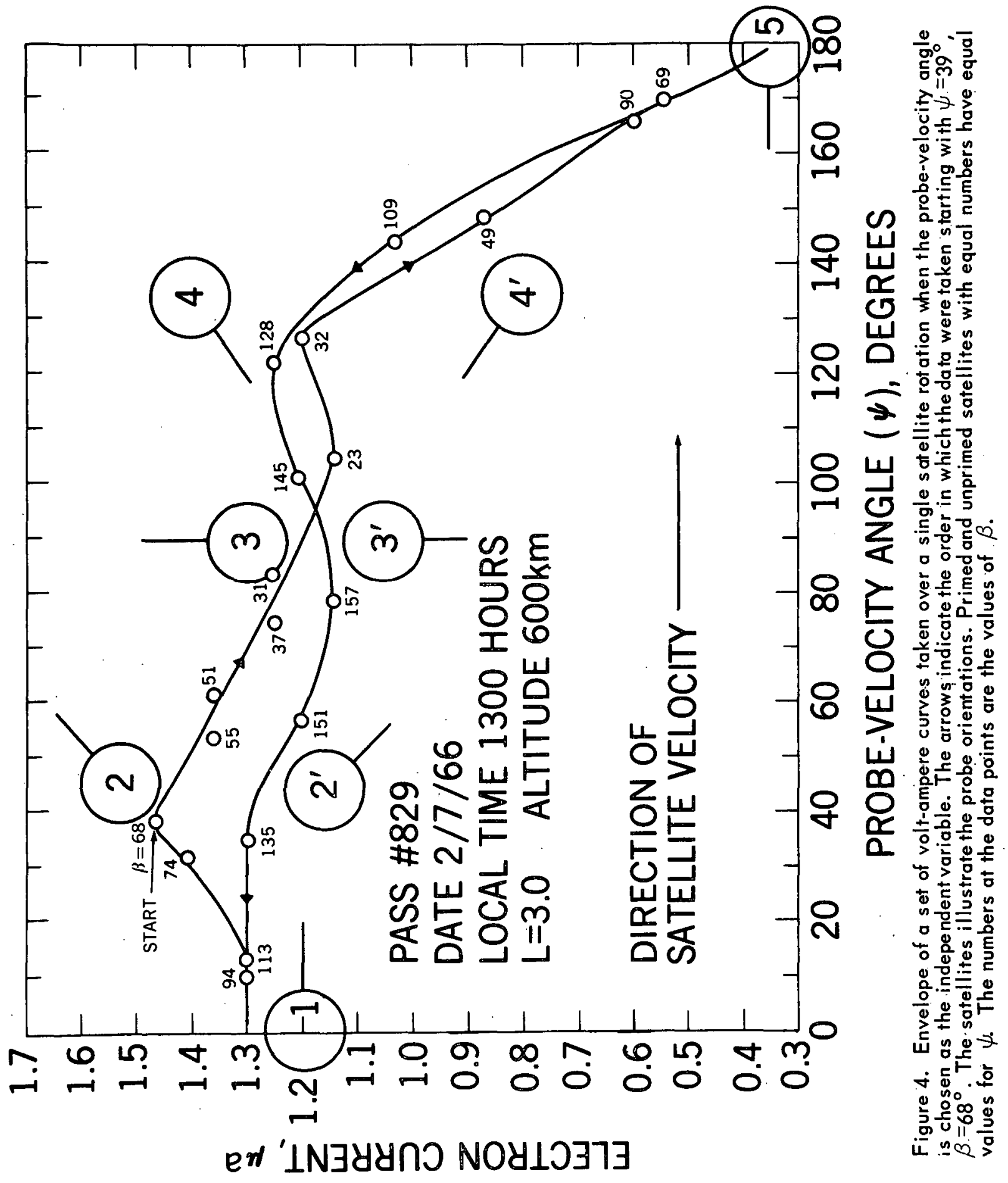




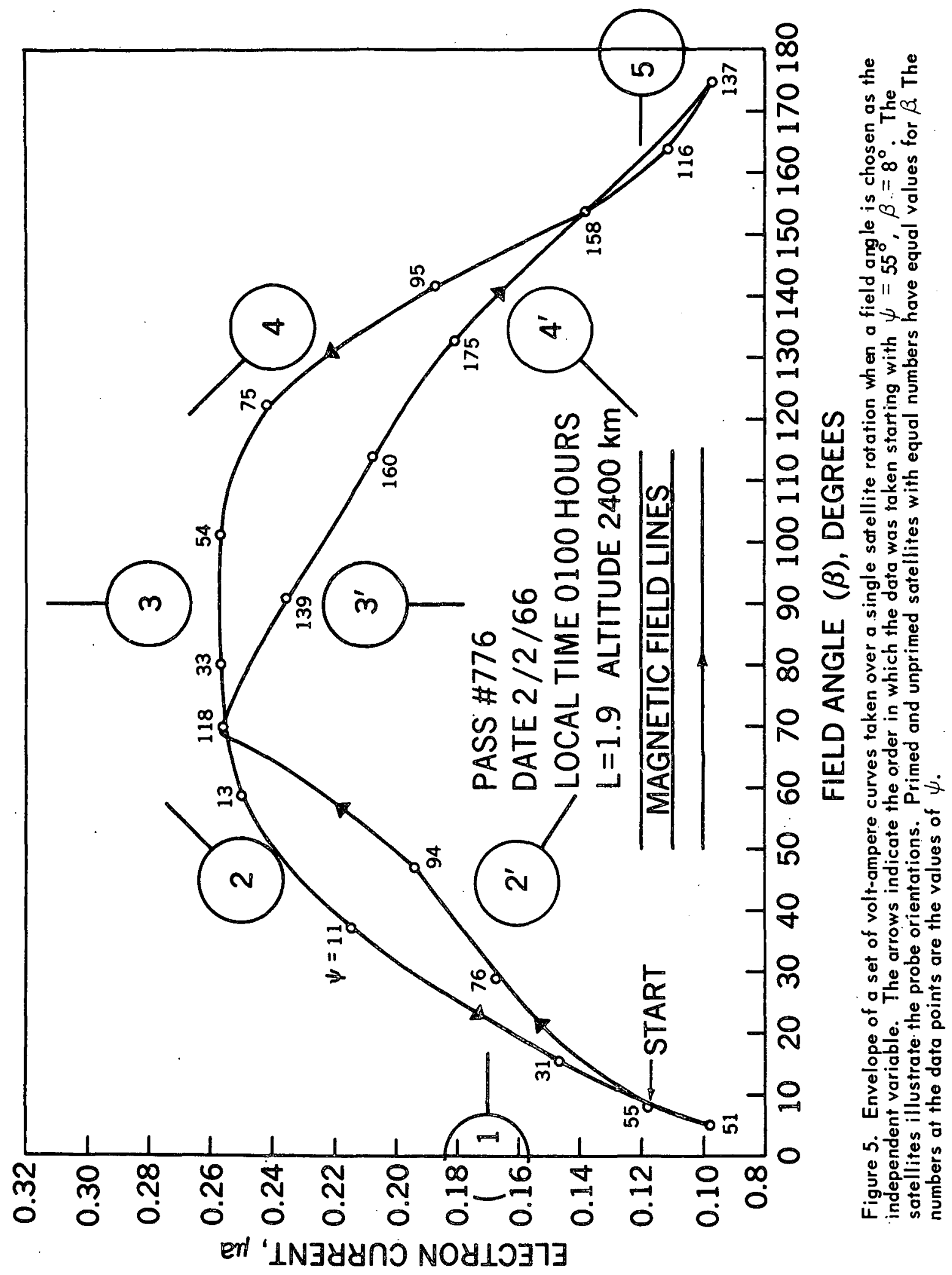




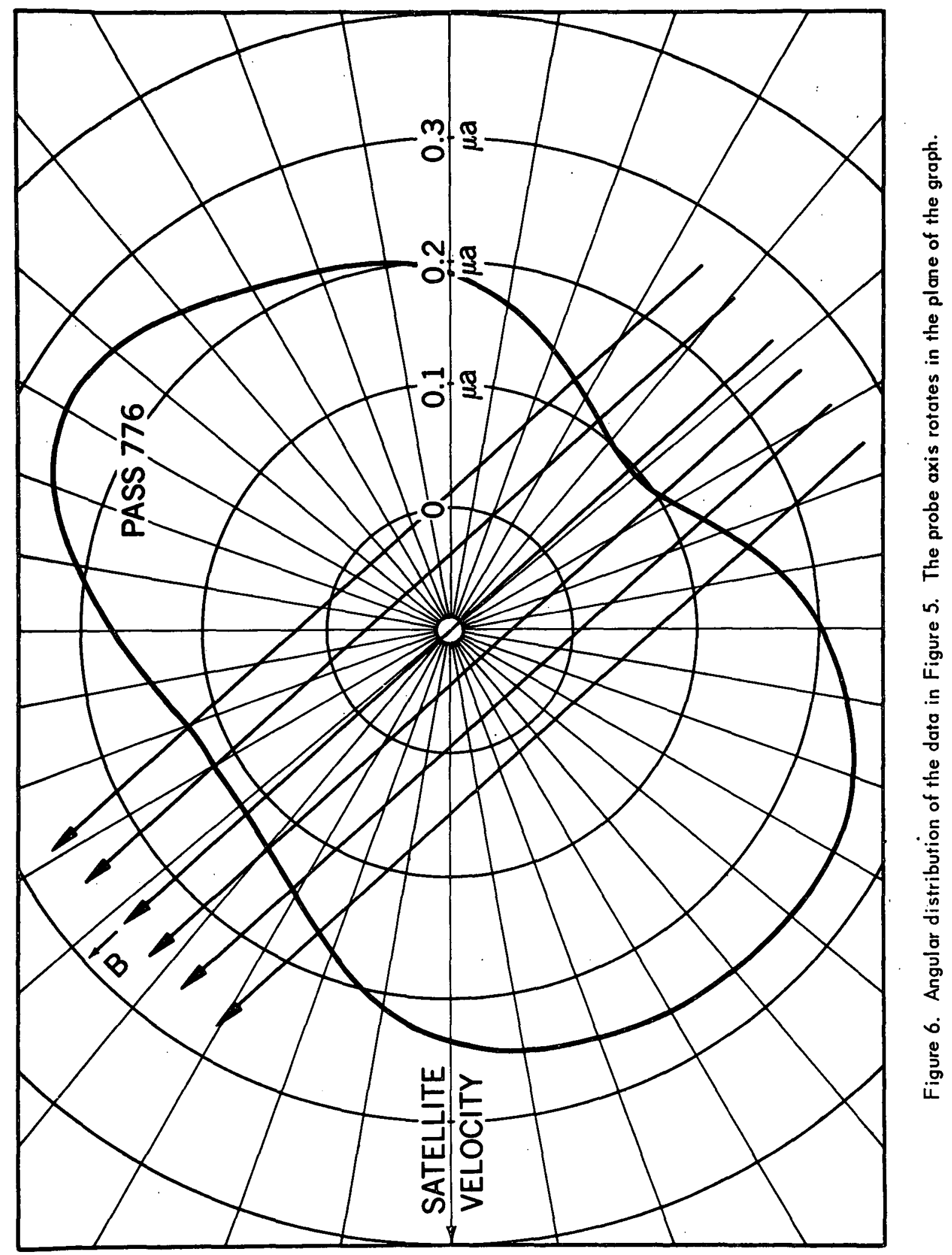




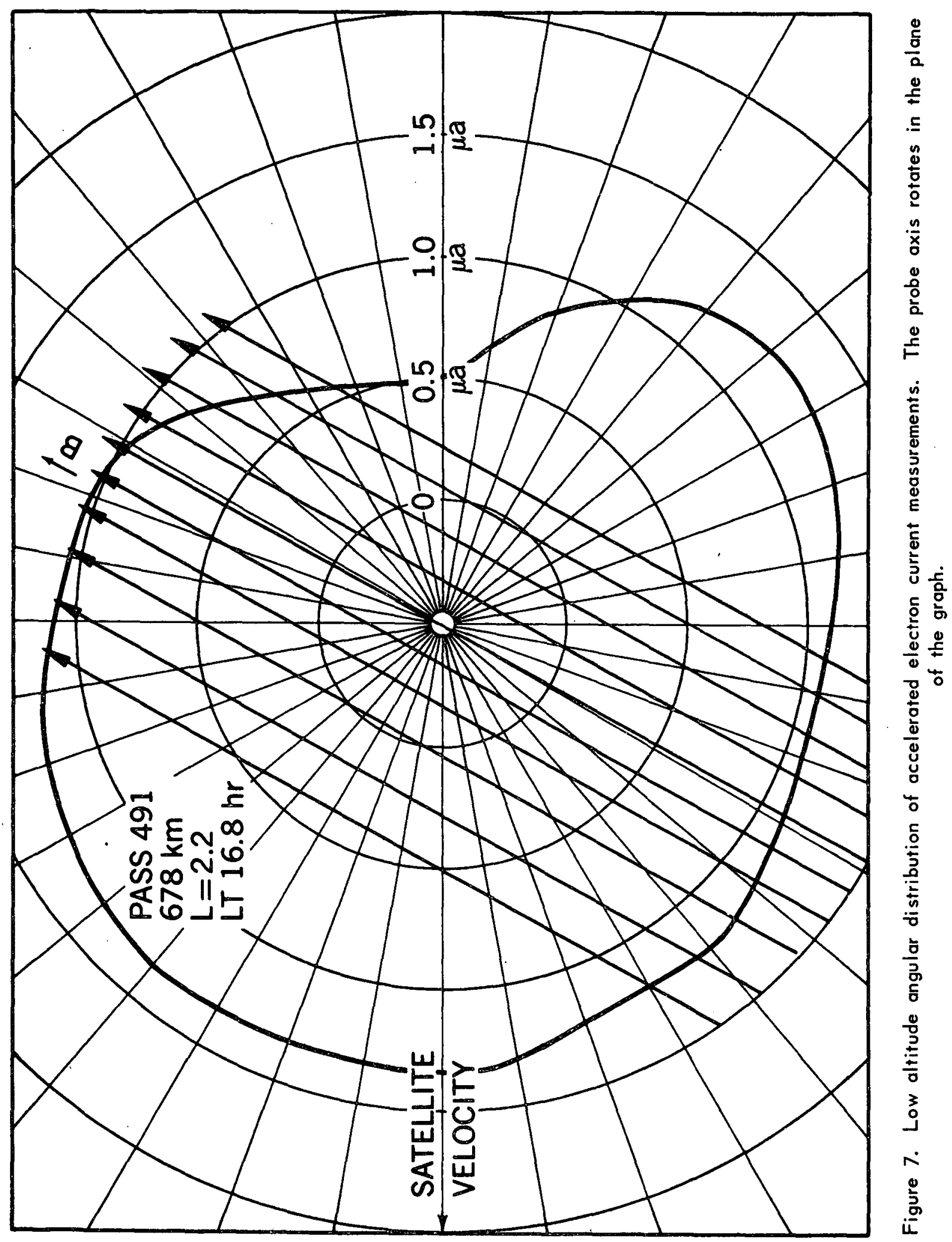




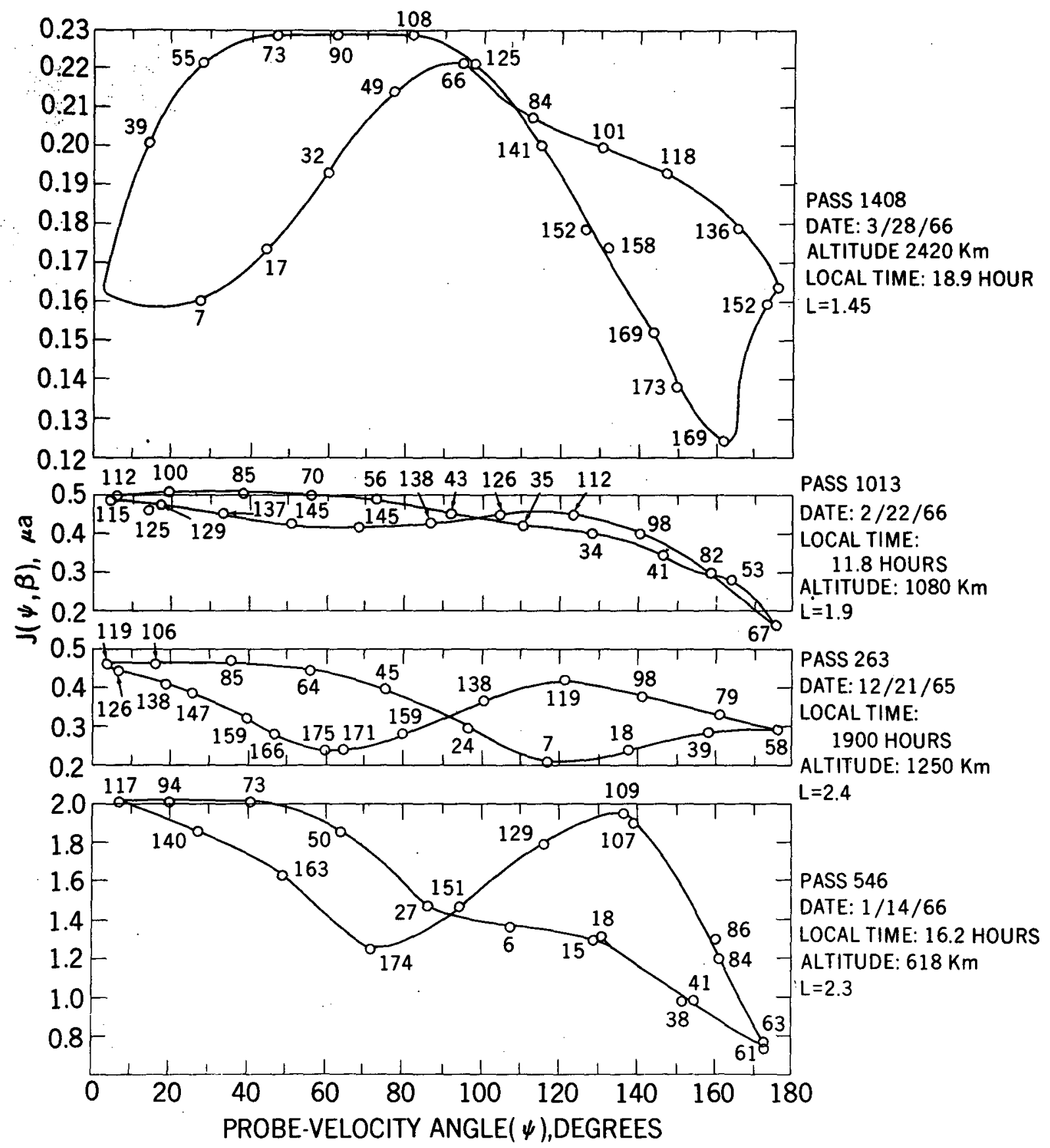

Figure 8. Electron current envelopes of volt-ampere curves taken during a satellite rotation with the probe-velocity angle chosen as the independent variable. The numbers at the data points give the value of the field angle. 


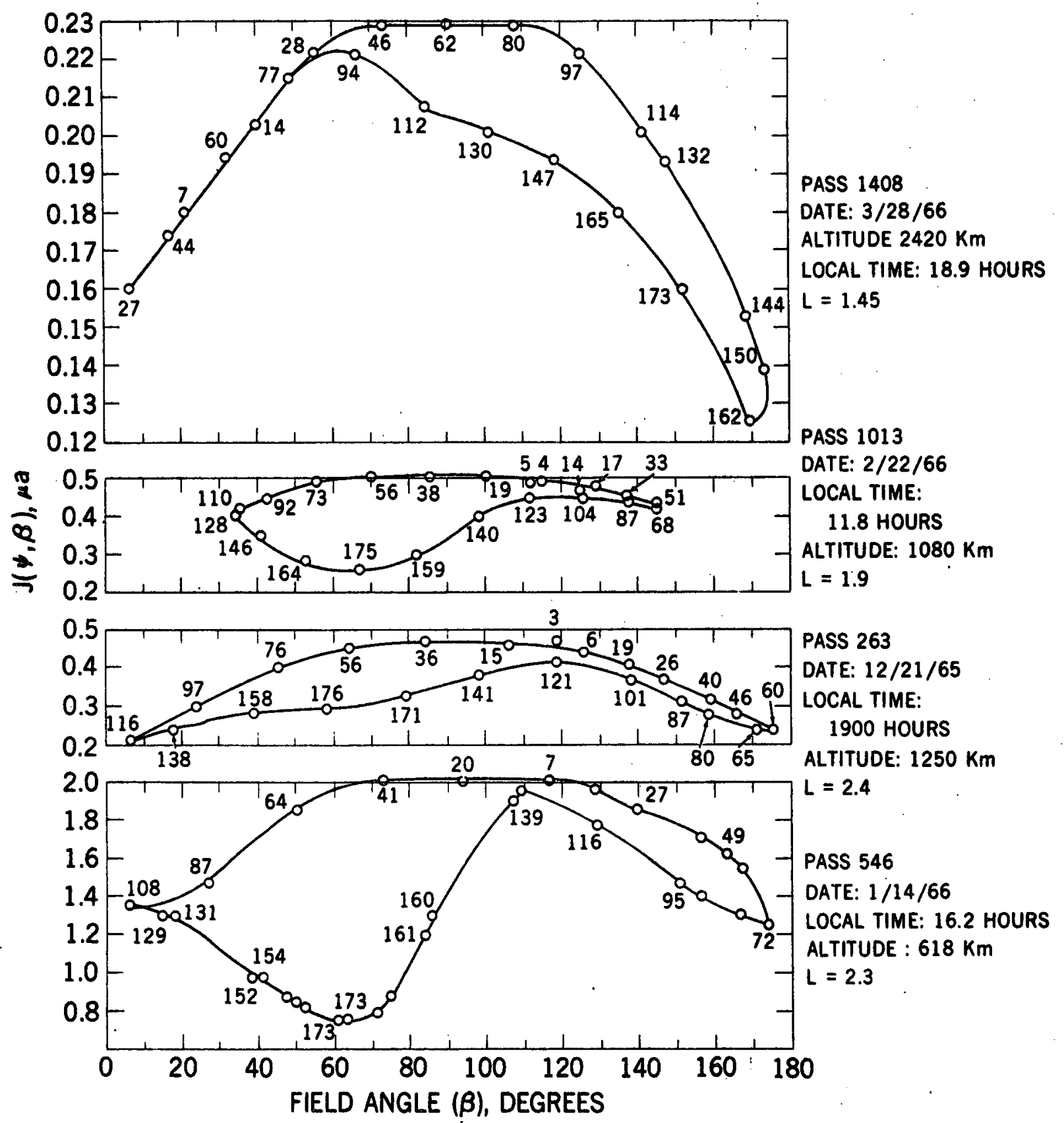

Figure 9. Electron current envelopes of the same data as Figure 8 with a field angle chosen as the independent variable. The numbers at the data points give the volue of the probe-velocity angle. 


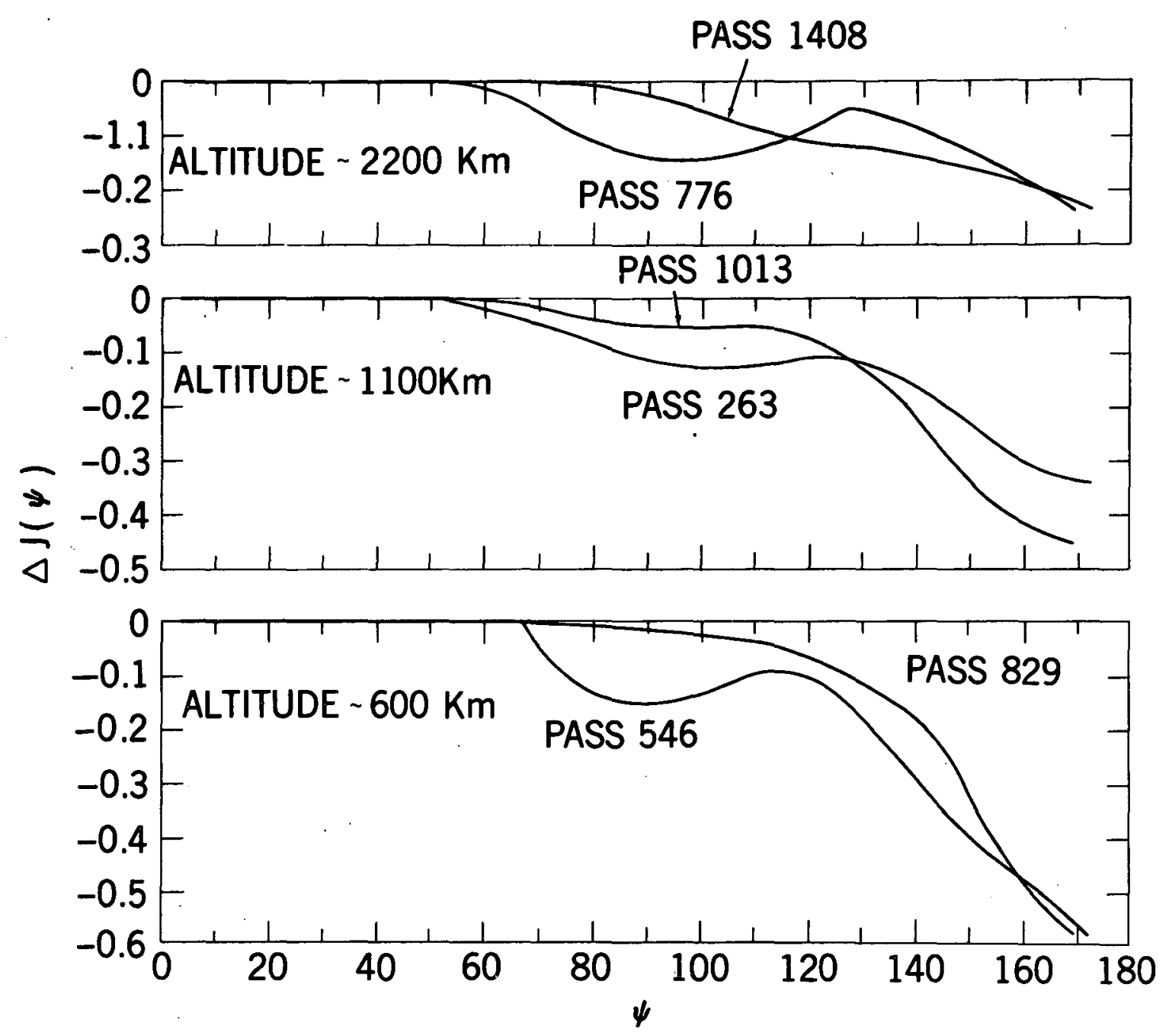

Figure 10. Normalized $\psi$ dependent component of total current modulations for the data of Figures 4 and 8. 


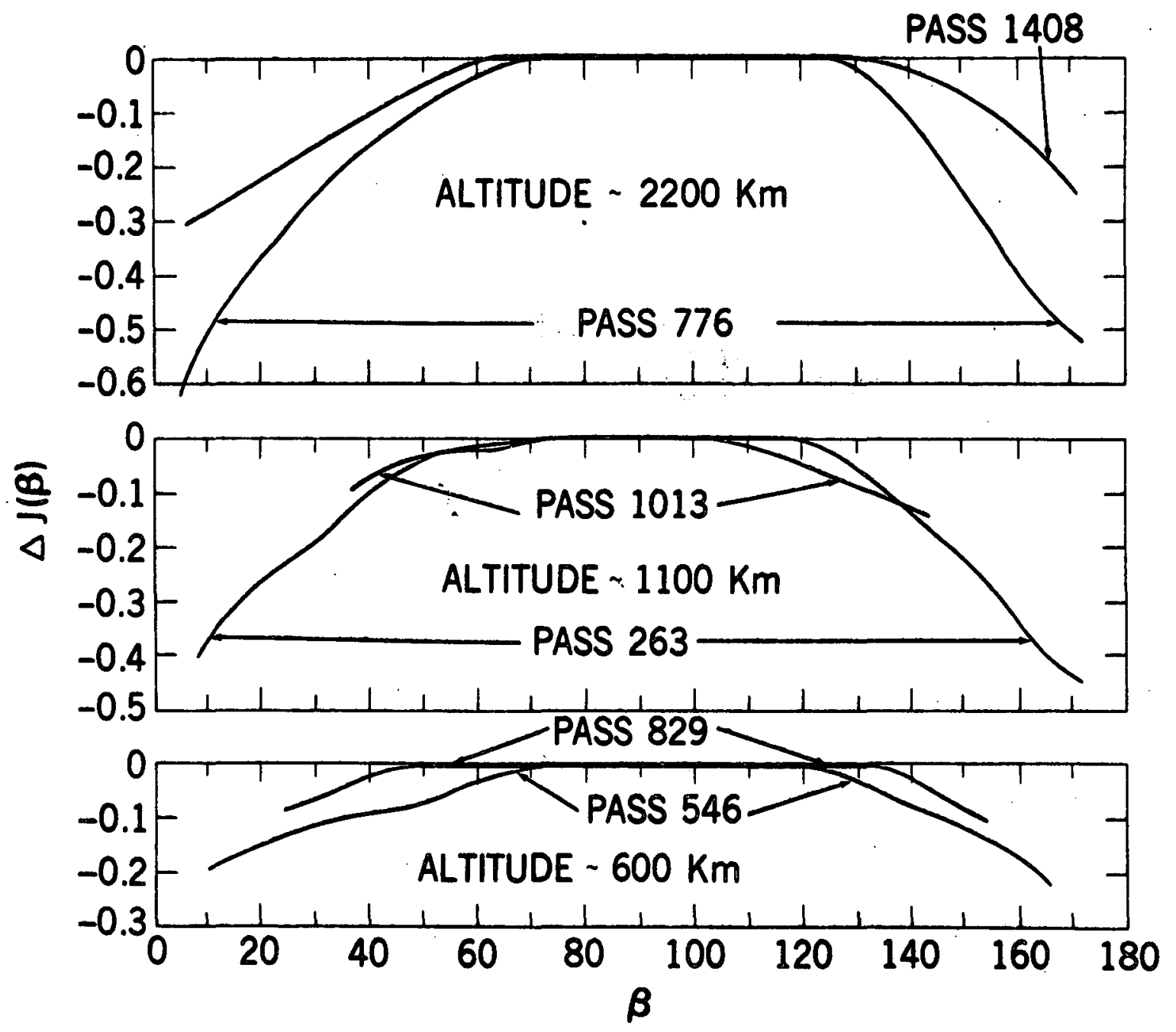

Figure 11. Normalized $\beta$ dependent component of total current modulations for the data of Figures 5 and 9. 